\title{
WHEN ARE REES CONGRUENCES PRINCIPAL?
}

\author{
C. M. REIS
}

(Communicated by Thomas H. Brylawski)

\begin{abstract}
Let $\rho_{I}$ be the Rees congruence modulo the ideal $I$ of the free monoid $X^{*}$. In this short note we give necessary and sufficient conditions, in terms of the partial order induced by division on the complement of $I$, for $\rho_{I}$ to be principal. In particular, we prove that if $I$ is principal, so is $\rho_{I}$.
\end{abstract}

\section{INTRODUCTION}

Let $X$ be a finite alphabet with at least two letters and $X^{*}$ the free monoid on $X$. Given a language $L$ on $X$, the principal congruence determined by $L$, and denoted $P_{L}$, is defined by: $u \equiv v\left(P_{L}\right)$ if the following is valid: for all $x, y \in X^{*}, x u y \in L$ if and only if $x v y \in L$. Recall that $L$ is a union of $P_{L}$-classes and that $P_{L}$ is the coarsest congruence saturating $L$. A congruence $\rho$ on $X^{*}$ is said to be principal if $\rho=P_{L}$ for some $L \subseteq X^{*}$. If $I$ is an ideal of $X^{*}$, the Rees congruence $\rho_{I}$ is defined by: $u \equiv v\left(\rho_{I}\right)$ if either $u, v \in I$ or $u=v$ when $u, v \notin I$.

In this short note we find necessary and sufficient conditions for the Rees congruence $\rho_{I}$ to be principal and deduce that the Rees congruence modulo a prime ideal is principal as is the Rees congruence modulo a principal ideal.

As for notation, the length of an element $w \in X^{*}$ (called a word) will be denoted by $|w|$ and the complement of a language $L$ over $X$ will be denoted by $\bar{L}$.

As a general reference we recommend G. Lallement's book [2].

\section{MAIN RESUltS}

Definition 1. (a) Define a partial order $\leq$ on $X^{*}$ by $u \leq v$ if $v=p u q$ for some $p, q \in X^{*}$.

(b) A set of incomparable elements relative to the partial order in (a) is called an infix code [3].

Notation. If $L$ is a language over $X$, the sets $\left\{u \in X^{*} \mid u \leq l\right.$ for some $l \in L\}$ and $\left\{u \in X^{*} \mid l \leq u\right.$ for some $\left.l \in L\right\}$ will be denoted by $(L]$ and $[L)$, respectively.

Received by the editors July 30, 1988 and, in revised form, October 12, 1988.

1980 Mathematics Subject Classification (1985 Revision). Primary 20M05; Secondary 20 M35. 
Lemma 2. Let I be an ideal of $X^{*}$ and $L$ a language over $X$. If $L \subseteq I$, then $[L) \subseteq I$ while if $L \subseteq \bar{I}$, the complement of $I$, then $(L] \subseteq \bar{I}$.

Proof. Clear from the definitions.

Definition 3. A language $\mathscr{C}$ over $X$ is a chain if any two words of $\mathscr{C}$ are comparable. A chain $\mathscr{C}$ is said to be maximal in $T$, where $T$ is a language over $X$, if $\mathscr{C}$ is contained in $T$ and is not properly contained in any chain contained in $T$.

A simple application of Zorn's lemma proves that, given a language $T$ over $X$ and $u \in T$, there exists a chain $\mathscr{C}$ maximal in $T$ and containing $u$.

Lemma 4. Let $I$ be an ideal of $X^{*}$. Then every chain $\mathscr{C}$ maximal in $\bar{I}$ has the property that, for any $x \in \mathscr{C}$ and any natural number $n \leq|x|$, there exists $y \in \mathscr{C}$ with $|y|=n$.

Proof. Let $m=|x|$. If $m=1$, the lemma is valid. Assume the lemma is true for a word of length $m-1$ belonging to $\mathscr{C}$ and let $|x|=m>1$. Let $x_{f}$ and $x_{l}$ be the words obtained by deleting the first and last letters of $x$, respectively. Clearly $(x]=\{x\} \cup\left(x_{f}\right] \cup\left(x_{l}\right]$, whence, since $(x] \subseteq \bar{I}$ by Lemma 2 and $\mathscr{C}$ is maximal, it follows that either $x_{f}$ or $x_{l}$ is in $\mathscr{C}$. But $\left|x_{f}\right|=\left|x_{l}\right|=m-1$ and thus by the induction hypothesis, given a natural number $n \leq m-1$, there is a word in $\mathscr{C}$ of length $n$. The result now follows by induction.

The following is the main result.

Theorem 5. Let $I$ be an ideal of $X^{*}$. Then $\rho_{I}$ is principal iff $\bar{I}$ contains at most one word which is maximal in $\bar{I}$.

Proof. Suppose first that $\bar{I}$ contains no maximal words. If $\bar{I}=\varnothing$, then $I=X^{*}$ and $\rho_{I}$ is clearly principal. Thus assume $\bar{I} \neq \varnothing$ and let $\bar{I}=\left\{x_{1}, x_{2}, \ldots\right\}$. Since $\bar{I}$ has no maximal words, each $x_{i}$ belongs to an infinite chain $C_{i}$ maximal in $\bar{I}$. By Lemma 4 , given any natural number $n$, there exists a word $w \in C_{i}$ with $|w|=n$. Let $p_{1}<p_{2}<p_{3} \cdots$ be the sequence of primes. For each $C_{i}$, choose a subchain $D_{i}=\left\{z_{1}^{(i)} \leq z_{2}^{(i)} \leq \cdots\right\}$ such that $\left|z_{j}^{(i)}\right|=2^{p_{j}^{i}}$ and let $L=\bigcup_{i} D_{i}$. Let $u, v \in L$ and let $|u|=2^{p_{s}^{i}},|v|=2^{p_{t}^{j}}$. If $|u|=|v|$, then $p_{s}^{i}=p_{t}^{j}$ whence $p_{s}=p_{t}$ and $i=j$. Therefore $s=t$ and $i=j$ whence $u=v$. Thus no two words of $L$ can have the same length. Let $u, v \in L, u \neq v$. Then $|u|=2^{s},|v|=2^{t}$ with $s>t$, say. Now $u \in D_{i}$ for some $i$, whence there exist $x, y \in X^{*}$ such that $x y \neq 1$ and $x u y \in D_{i} \subseteq L$. Let $|x u y|=2^{l}$. Then $|x|+|y|=2^{l}-2^{s}, l>s>t$. But $|x v y|=2^{l}-2^{s}+2^{t}=2^{t}\left(2^{l-t}-2^{s-t}+1\right)$ and hence, since $2^{l-t}-2^{s-t}+1$ is odd, it follows that $x v y \notin L$. Therefore $u \not \equiv v\left(P_{L}\right)$ and hence every $P_{L}$-class contained in $L$ is a singleton class. Let $w \in \bar{I}$. Then $w \in C_{i}$ for some $i$ and thus there exist $p, q \in X^{*}$ such that $p w q \in L$. But, denoting the $P_{L}$-class of a word $x$ by $[x]_{L}$, we have $p[w]_{L} q \subseteq$ $[p w q]_{L}=\{p w q\}$ whence $\left|[w]_{L}\right|=1$. Moreover, $\rho_{I}$ saturates $L$ and so $I$ 
is contained in a $P_{L}$-class since $P_{L}$ is the coarsest congruence saturating $L$. Since the $P_{L}$-classes in $\bar{I}$ are singletons, it follows that $I$ is a $P_{L}$-class and hence $P_{L}=\rho_{I}$.

Assume now that $\bar{I}$ contains exactly one maximal word, say $w$. If $\bar{I}=$ $(w]$, then clearly $\rho_{I}=P_{\{w\}}$. Thus assume that $(w] \neq \bar{I}$. Each word $x_{i}$ of $\bar{I} \backslash(w]=\left\{x_{1}, x_{2}, \ldots\right\}$ belongs to an infinite chain $C_{i}$ maximal in $\bar{I}$. Choose the subchains $D_{i}$ of $C_{i}$ as above and let $L=\left(\bigcup_{i} D_{i}\right) \cup\{w\}$. Let $u, v \in L \backslash\{w\}$, $u \neq v$. Then $|u|=2^{s},|v|=2^{t}, s>t$ and there exist $x, y \in X^{*}$ such that $|x|+|y|>|w|$ and $x u y \in L \backslash\{w\}$. Arguing as before and since $|x|+|y|>|w|$, it follows that $x v y \notin L$. Therefore $u \not \equiv v\left(P_{L}\right)$. Let $u \in L \backslash\{w\}$. Then $u \not \equiv w\left(P_{L}\right)$ since the only context of $w$ is $(1,1)$. Thus the $P_{L}$-classes contained in $L$ are singleton classes. As above, since $\left(X^{*} u X^{*}\right) \cap L \neq \varnothing$ for all $u \in \bar{I}$, it follows that $\rho_{I}=P_{L}$.

Conversely, suppose $\bar{I}$ contains more than one maximal word, say $u, v$ with $u \neq v$. Suppose there exists $L$ with $\rho_{I}=P_{L}$. Since $P_{L}$ saturates $L$, it follows that either $I \cap L=\varnothing$ or $I \subseteq L$. Assume, therefore, without loss of generality, that $L \subseteq \bar{I}$. Since $u$ and $v$ are maximal in $\bar{I}, x u y \in \bar{I}$ or $x v y \in \bar{I}$ implies $x y=1$. If $u \notin L$ (resp. $v \notin L)$, then $u \equiv w P_{L}$ (resp. $v \equiv w P_{L}$ ) for all $w \in I$, a contradiction. Hence $u, v \in L$ and $u \equiv v P_{L}$. This contradiction shows that $\rho_{I}$ cannot be principal.

We now obtain a necessary and sufficient condition for a Rees congruence not to be principal in terms of the minimal generating set of the ideal.

Definition 6 [1]. Let $I$ be an ideal of $X^{*}$. Then the set of minimal words of $I$ is called the infix root of $I$ and is denoted by $r(I)$.

Clearly $I=[r(I))=X^{*} r(I) X^{*}$. It is also obvious that $r(I)$ is the minimal generating set of $I$.

Definition 7. The word $u$ is a prefix (resp. suffix) of $w$ if $w=u x$ (resp. $w=x u$ ) for some $x \in X^{*}$.

Notation. For a given language $L$, let $\mathscr{P}(L)=\{u \mid u \neq 1$ and $u$ is a prefix of some word in $L\}, \mathscr{S}(L)=\{u \mid u \neq 1$ and $u$ is a suffix of some word in $L\}$.

Corollary 8. Let $I$ be an ideal of $X^{*}$. Then $\rho_{I}$ is not principal iff there exist distinct words $u$ and $v$ in $\bar{I}$ such that

$$
\begin{aligned}
|\mathscr{P}(X u) \cap r(I)| & =|\mathscr{S}(u X) \cap r(I)| \\
& =|\mathscr{P}(X v) \cap r(I)| \\
& =|\mathscr{S}(v X) \cap r(I)| \\
& =|X| .
\end{aligned}
$$

Proof. If $\rho_{I}$ is not principal, by Theorem $5 \bar{I}$ contains two distinct words $u$ and $v$ which are maximal in $\bar{I}$. Clearly $(u X) \cup(X u) \cup(v X) \cup(X v) \subseteq I$. Let $a \in X$. Then there exists $w \in r(I)$ such that $w \leq a u$, whence $w=a u^{\prime}$ where $u^{\prime} u^{\prime \prime}=u$. 
Similar statements apply to $u a, a v$, and $v a$ and the necessity of the result follows.

Conversely, if the condition holds, $u$ and $v$ are maximal in $\bar{I}$ and thus by Theorem 4, $\rho_{I}$ is not principal.

Recall that an ideal $I$ of a semigroup $S$ is prime, if for all $a, b \in S, a S b \subseteq I$ implies $a \in I$ or $b \in I$. We are now able to prove the following.

Theorem 9. I is either a prime ideal or $|r(I)|<|X|$, then $\rho_{I}$ is principal. Hence, if $I$ is principal, $\rho_{I}$ is principal.

Proof. If $I$ is prime, then given $u, v \notin I$, there exists $x \in X^{*}$ such that $u x v \notin I$. Hence $\bar{I}$ contains no maximal word whence, by Theorem $5, \rho_{I}$ is principal. If $|r(I)|<|X|,|\mathscr{P}(X u) \cap r(I)|<|X|$ for all $u \in X^{*}$. Therefore, by Corollary $8, \rho_{I}$ is principal.

Remark. It can easily be proved that if $|X| \geq 3$ all principal ideals are prime. However, if $|X|=2, X^{*} u X$ is prime iff $u \notin a b^{+} \cup a^{+} b \cup b a^{+} \cup b^{+} a$ where $X=\{a, b\}$.

Definition 10. An infix code $L$ is said to be maximal if $L \cup\{w\}$ is not an infix code for any $w \in X^{*} \backslash L$.

Theorem 11. Let $X$ be an alphabet with more than one letter and let $I$ be an ideal of $X^{*}$ such that $r(I)$ is a finite maximal infix code. Then $\rho_{I}$ is not principal provided $r(I) \neq X$.

Proof. Since $r(I)$ is finite and maximal, $\bar{I}$ is also finite and hence contains words which are maximal in $\bar{I}$. Thus, by Theorem $5 \bar{I}$ contains exactly one maximal word $w$ if $\rho_{I}$ is principal. Clearly $\bar{I}=(w]$ and $\bar{I} \neq\{1\}$ since $r(I) \neq X$. By the maximality of $r(I)$, there exists $t \in r(I)$ such that $w \leq t$. Letting $t_{f}$ and $t_{l}$ denote the words obtained by deleting the first and last letters of $t$, respectively, we have $t_{f} \leq w \leq t$ and $t_{l} \leq w \leq t$. Hence $t_{f}=w=t_{l}$ whence $w=a^{s}, s>0$ for some $a \in X$ and $t=a^{s+1}$. It follows that each word of $r(I)$ is a power of the letter $a$. Hence $r(I)=\left\{a^{s+1}\right\}$, contradicting the maximality of the infix code $r(I)$. Hence $\rho_{I}$ is not principal.

The above theorem is not valid if $|r(I)|=\infty$ as the following example shows.

Example 12. Let $X=\{a, b\}$ and let $w=a_{1}^{n_{1}} a_{2}^{n_{2}} \cdots a_{s}^{n_{s}} \neq 1$ where $n_{i}>0$ and $a_{i} \neq a_{i+1}$ for all $i$. Then the skeleton of $w$, denoted $\operatorname{sk}(w)$, is the word $a_{1} a_{2} \cdots a_{s}$. Let

$$
\left.L=\left\{a b^{i} a^{j} b \mid i, j>0\right\} \cup\left\{b a^{i} b^{j} a \mid i, j>0\right\} \cup a^{2} b^{j} a^{2} \mid j>0\right\} \cup\left\{b^{2} a^{j} b^{2} \mid j>0\right\} .
$$

$L$ is clearly an infix code. That it is maximal is a consequence of the following: first, if $|\operatorname{sk}(w)| \leq 2$, clearly $w \in(L]$; if $|\operatorname{sk}(w)|=3, w=a^{i} b^{j} a^{k}$ or $w=$ $b^{i} a^{j} b^{k}$. In either case, if $i, k \geq 2, w \in[L)$, while if one of $i$ and $k$ is 1 , $w \in(L]$. If $|\mathbf{s k}(w)| \geq 4, w \in[L)$ thus proving that $L$ is indeed maximal infix. 
It is easy to check that

$$
\begin{aligned}
\overline{X^{*} L X^{*}}=\{w|| \operatorname{sk}(w) \mid \leq 2\} & \cup\left\{a b^{j} a^{k}, b a^{j} b^{k} \mid j, k>0\right\} \\
\cup & \left\{a^{i} b^{j} a, b^{i} a^{j} b \mid i, j>0\right\} \cup\{1\}
\end{aligned}
$$

and that no word of $\overline{X^{*} L X^{*}}$ is maximal in $\overline{X^{*} L X^{*}}$. By Theorem 5 , it follows that the Rees congruence modulo the ideal $X^{*} L X^{*}$ is principal.

Theorem 13. Let $I$ be an ideal of $X^{*}$ such that $\rho_{I}$ is principal and let $L \subseteq \bar{I}$ be a language with $\rho_{I}=P_{L}$. Let $F$ be a finite subset of $L$ not containing the maximal word of $\bar{I}$ (if such exists). Then $P_{L \backslash F}=\rho_{I}$.

Proof. Let $T=L \backslash F$ and let $u, v \in \bar{I}$. Assume first that $\bar{I}$ contains no maximal word. Then $u$ belongs to an infinite chain in $\bar{I}$. Hence there exist $p, q \in X^{*}$ such that $|p u q|$ and $|p v q|$ are greater than $\max \{|w| \mid w \in F\}$ and $p u q \in \bar{I}$. Let $u \equiv v\left(P_{T}\right)$. Then $p u q \equiv p v q\left(P_{T}\right)$. Let $x p u q y \in L$. Since $|x p u q y|>\max \{|w| \mid w \in F\}$, it follows that $x p u q y \in T$, whence $x p v q y \in$ $T \subseteq L$. Similarly, $x p v q y \in L$ implies $x p u q y \in L$, thus proving that $p u q \equiv$ $p v q\left(P_{L}\right)$. Hence $p u q=p v q$ and $u=v$. Therefore $\rho_{I}=P_{T}$. Suppose now that $\bar{I}$ contains a maximal word $y$ and let $u, v \in \bar{I}$. If one of $u$ and $v$ belongs to an infinite chain of $\bar{I}$, the same argument as above shows that $u \equiv v\left(P_{T}\right)$ implies $u=v$. Suppose therefore that neither word belongs to an infinite chain of $\bar{I}$. Then $u \leq y, v \leq y$ and thus there exist $p, q \in X^{*}$ such that $p u q=y$. Since $y \notin F$, it follows that $p u q \in T$. Hence, if $u \equiv v\left(P_{T}\right)$, then $p v q \in T$. If $p v q=y$, then $u=v$. Suppose if possible that $p v q \neq y$.

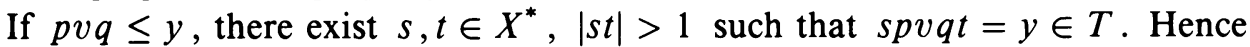
spuqt $=$ syt $\in T \subseteq \bar{I}$, a contradiction since $y$ is maximal in $\bar{I}$. Thus $y$ and $p v q$ are incomparable. Therefore $p v q$ belongs to an infinite chain of $\bar{I}$. An argument similar to the one given above proves $p u q=p v q=y$, a contradiction. Thus $P_{T}$ is equality on $\bar{I}$. Clearly $I$ is a $P_{T}$-class, whence $P_{T}=\rho_{I}$.

We conclude by giving an example to show that the above theorem is not valid if $\bar{I}$ contains a maximal word $y$ and $y \in F$.

Example 14. Let $X=\{a, b\}, \bar{I}=a^{*} \cup\left(a^{2} b^{2}\right]$. Then $\rho_{I}=\rho_{L}$ where $L=$ $\left\{a^{2^{n}} \mid n \geq 1\right\} \cup\left(a^{2} b^{2}\right]$. However $P_{L \backslash\left\{a^{2} b^{2}\right\}} \neq \rho_{I}$. In fact $a b^{2} \equiv a^{2} b\left(P_{L \backslash\left\{a^{2} b^{2}\right\}}\right)$ and $a b^{2}, a^{2} b \in \bar{I}$.

\section{REFERENCES}

1. Y. Q. Guo, H. J. Shyr and G. Theirrin, $Q f$-disjunctive languages, Papers on automata and language VII, Department of Mathematics, Karl Marx University of Economics, Budapest, 1985, pp. 413-440.

2. G. Lallement, Semigroups and combinatorial applications, Wiley-Interscience, New York, 1979.

3. C. M. Reis, Infix congruences on a free monoid, Trans. Amer. Math. Soc. vol. 310, Number 2, Dec. 1988.

Mathematics Department, University of Western Ontario, London, Ontario N6A 5B7, CANADA 